\title{
A ENFERMAGEM E A ATENÇÃO PRIMÁRIA À SAÚDE FRENTE À PANDEMIADE COVID-19 NO BRASIL
}

\author{
NURSING AND PRIMARY HEALTH CARE IN FRONT OF THE COVID- \\ 19PANDEMIC IN BRAZIL
}

\author{
Karén Kelyany Duarte Costa ${ }^{1}$ \\ Anny Isabelly Medeiros de Goés ${ }^{2}$ \\ Francilene Maciel Ferreira Silva ${ }^{3}$ \\ Laura Aires Cavalcante Leite ${ }^{4}$ \\ Renata Ferreira de Araújo ${ }^{5}$
}

RESUMO: OBJETIVO: Conhecer a atuação da enfermagem e da atenção primária à saúde, frente à pandemia de COVID-19 no Brasil. MÉTODO: revisão integrativa realizada no mês de fevereiro de 2021, com busca nas seguintes bases de dados Scientific Electronic Library Online (SciELO), Medical Literature Analysis and Retrieval System Online (Medline) e Google Acadêmico. Foram utilizados como critérios de inclusão artigos na língua portuguesa e inglesa, disponíveis na integra e de forma gratuita e àqueles que respondessem a questão norteadora: Como a enfermagem e a atenção primária à saúde atuam frente à pandemia de COVID-19 no Brasil? Foram excluídas as produções incompletas e que não atendiam ao objetivo do estudo. RESULTADOS: a amostra resultou em seis artigos que versam sobre: a APS como sendo porta de entrada para os pacientes suspeitos e/ou confirmados de Covid-19 e a Enfermagem com um papel importante através da organização da unidade e da aplicação de protocolos estabelecidos para o momento. CONCLUSÃO: evidencia-se a necessidade do reconhecimento da enfermagem e do papel da APS, visto que toda a rede de atenção foi fragilizada por falta de recursos materiais e financeiros e pelo óbito de muitos profissionais.

Palavras chave: Atenção Primária à Saúde. Coronavirus. Cuidados de Enfermagem. Pandemia.

\footnotetext{
${ }^{1}$ Graduanda no curso de Enfermagem pela Universidade Estadual da Paraíba - UEPB;

${ }^{2}$ Graduanda no curso de Enfermagem pela Universidade Estadual da Paraíba - UEPB;

${ }^{3}$ Graduanda no curso de Enfermagem pela Universidade Estadual da Paraíba - UEPB;

${ }^{4}$ Graduanda no curso de Enfermagem pela Universidade Estadual da Paraíba - UEPB;

${ }^{5}$ Graduanda no curso de Enfermagem pela Universidade Estadual da Paraíba - UEPB.
} 
ABSTRACT: OBJECTIVE: To learn about the role of nursing and primary health care in the face of the COVID-19 pandemic in Brazil. METHOD: integrative review carried out in February 2021, searching the following databases Scientific Electronic Library Online (SciELO), Medical Literature Analysis and Retrieval System Online (Medline) and Google Scholar. Inclusion criteria were articles in Portuguese and English, available in full and free of charge, and to those who answered the guiding question: How do nursing and primary health care work in the face of the COVID-19 pandemic in Brazil? Incomplete productions that did not meet the objective of the study were excluded. RESULTS: the sample resulted in six articles that deal with: PHC as a gateway for suspected and / or confirmed patients from Covid-19 and Nursing with an important role through the organization of the unit and the application of protocols established for the moment. CONCLUSION: there is a need for recognition of nursing and the role of $\mathrm{PHC}$, since the entire care network has been weakened by the lack of material and financial resources and the death of many professionals.

Keywords: Primary Health Care. Coronavirus. Nursing care. Pandemic. 


\section{INTRODUÇÃO}

A pandemia ocasionada pelo novo Coronavírus colocou em evidência a crise na saúde pública de diversos países, tornando necessário o planejamento para o enfrentamento mundial que requer essa situação de emergência. Este vírus, nomeado "Severe Acute Respiratory Syndrome Coronavirus 2" - SARS-CoV-2, produz a doença classificada como Coronavirus Disease 2019 - COVID-19, sendo agente causador de uma série de casos de infecções, em especial, as respiratórias. Esta patologia é uma ameaça urgente e disseminada, cujas características clínicas e epidemiológicas ainda estão sendo documentadas (SANCHEZ et al., 2020).

Nesse sentido, a Atenção Primária à Saúde (APS) ou Atenção Básica (AB), por meio da Estratégia Saúde da Família (ESF), é a porta de entrada do Sistema Único de Saúde (SUS). Durante surtos e epidemias, a APS/ESF tem papel fundamental na resposta global à doença em questão. A APS/ESF oferece atendimento resolutivo, além de manter a longitudinalidade e a coordenação do cuidado em todos os níveis de atenção à saúde, com grande potencial de identificação precoce de casos graves que devem ser manejados em serviços especializados(TOCANTIS, 2020).

Em sua vocação para a ação comunitária, a APS pode ampliar a capacidade de resposta local não só para reduzir a disseminação da infecção, como também para amenizar os efeitos sociais e econômicos das medidas de distanciamento social. Por exemplo, por intermédio das mídias sociais e rádios comunitárias, as equipes de saúde da família (EqSF) podem orientar a população sobre as formas de contágio e informar sobre os canais de teleatendimento (DAUMAS, 2020).

Seguindo na esteira desse conhecimento, em meio a esta magnitude epidemiológica estabelecida pela COVID-19, os trabalhadores da saúde, sobretudo os de Enfermagem, atenderam o chamamento para assumirem a linha de frente, atuando na coordenação do cuidado à população nos diferentes pontos da Rede de 
Atenção à Saúde (RAS), na vigilância e monitoramento de casos sintomáticos respiratórios no lar e na comunidade, no território adscrito por tessituras geográficas e de sentidos e sentimentos na APS, bem como nos espaços de maior densidade tecnológica (SILVA et al.,2020).

O papel da enfermagem no cuidado às pessoas doentes ou com suspeita de infecção por COVID-19 tem sido ressaltado em termos da necessária competência técnica e humana para o cuidado ágil e seguro. Mas nas últimas semanas fica cada vez mais claro que a atuação da enfermagem como mediadora entre famílias e pessoas internadas com COVID-19 é essencial para diminuir o sentimento de solidão e medo dos pacientes isolados e cuidados por profissionais de quem mal se vislumbram os olhos. Essa competência humanística não se dissocia da competência ético-política e do papel pedagógico da prática da enfermagem (DAVID et al., 2021).

Um surto global requer a participação ativa da força de trabalho de Enfermagem no atendimento clínico, educação/formação e no compartilhamento de informações sobre saúde pública e políticas. O engajamento e protagonismo dos enfermeiros frente à pandemia no desenvolvimento do cuidado ou ações de controle para sua mitigação os colocam como atores-chave no fim do surto, por conta de "seu compromisso, dedicação, espírito de sacrifício, treinamento e responsabilidade estão além de qualquer dúvida e nesta crise, como em outras anteriores, o estão demonstrando de maneira ampla (SILVA et al.,2020).

Portanto, faz-se necessário que a sociedade reconheça os esforços existentes entre os profissionais enfermeiros e, consequentemente, reconheçam o empenho da APS enquanto rede orientadora de estratégias de cuidado integral e do combate à precarização da saúde. Destarte, o objetivo do estudo consistiu em conhecer a atuação da enfermagem e da atenção primária à saúde, frente à pandemia de COVID-19 no Brasil. 


\section{MÉTODO}

Trata-se de uma revisão integrativa de literatura no qual se buscou conhecer a atuação de enfermagem frente à pandemia de COVID-19, destacando a importância da atenção primária à saúde no cenário atual.

Realizou-se um levantamento de dados bibliográficos no período de fevereiro de 2021, através do banco de dados BVS - Biblioteca Virtual em Saúde e no Google Acadêmico. A amostra foi composta por seis produções cientificas que foram analisadas mediante os critérios de inclusão, exclusão e a questão norteadora.

Quadro 1. Descrição do protocolo de busca da pesquisa.

\section{A ENFERMAGEM E A ATENÇÃO PRIMÁRIA À SAÚDE FRENTE À PANDEMIA DE COVID-19 NO BRASIL}

\begin{tabular}{|c|c|}
\hline OBJETIVO & $\begin{array}{l}\text { onhecer a atuação da enfermagem e da atenção primária à } \\
\text { aúde,frente à pandemia de COVID-19 no Brasil. }\end{array}$ \\
\hline $\begin{array}{l}\text { UESTÃO } \\
\text { ORTEADORA }\end{array}$ & $\begin{array}{l}\text { Como a enfermagem e a atenção primária à saúde atuam } \\
\text { frente àpandemia de COVID-19 no Brasil? }\end{array}$ \\
\hline FONTE DOSDADOS & $\begin{array}{l}\text { BVS - Biblioteca Virtual em Saúde: } \\
\text { Lilacs (Literatura Latino-americana e do Caribe em Ciências } \\
\text { da Saúde), Scielo (Scientific Electronic Library Online), } \\
\text { Medline (Medical Literature Analysis and Retrieval System } \\
\text { Online). } \\
\text { Google Acadêmico. }\end{array}$ \\
\hline DESCRITORES & $\begin{array}{l}\text { Português: cuidados de enfermagem, coronavírus, } \\
\text { pandemia,atenção primária à saúde. } \\
\text { Inglês: Nursing care, coronavirus, pandemics, primary health } \\
\text { care. }\end{array}$ \\
\hline EXP & $\begin{array}{l}\text { Português: } \\
1 . \quad \text { ("cuidados de enfermagem") AND ("coronavírus"). } \\
2 . \quad \text { ("cuidados de enfermagem") AND ("coronavírus") } \\
\text { AND("atenção primária à saúde"). } \\
3 . \quad \text { ("coronavírus") AND ("atenção primária à saúde"). } \\
\text { Inglês: } \\
\text { 1. ("Nursing care") AND (“coronavirus"). } \\
\text { 2. ("Nursing care") AND ("coronavirus") AND ("Primary } \\
\text { HealthCare"). } \\
\text { 3. ("coronavirus") AND ("Primary Health Care"). }\end{array}$ \\
\hline
\end{tabular}




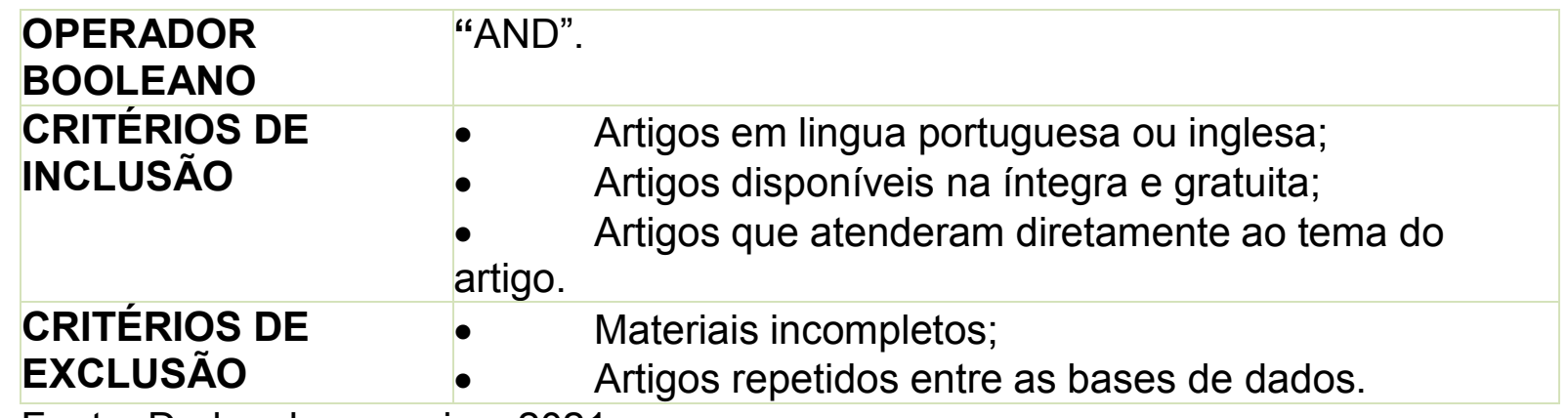

Fonte: Dados da pesquisa, 2021.

\section{RESULTADOS}

Considerou-se para o estudo a amostra de 06 artigos (Tabela 1), ressaltando a aplicação dos critérios de exclusão e da leitura exploratória. Os artigos incluídos constaram referências relevantes à questão norteadora do estudo e, dessa forma, as principais informações são apresentadas no Quadro 2.

Tabela 1. Banco de dados, quantidade de artigos encontrados, selecionados e incluídos.

\begin{tabular}{cccc}
\hline Banco de dados & Encontrados & Selecionados & Incluídos \\
\hline SCIELO & 31 & 5 & 2 \\
LILACS & 16 & 0 & 0 \\
MEDLINE & 8 & 3 & 1 \\
Google Acadêmico & 321 & 11 & 3 \\
\hline Total & 376 & 19 & 6 \\
\hline
\end{tabular}

Fonte: Dados da pesquisa, 2021. 
Quadro 2. Síntese dos artigos incluídos no estudo, através da busca nas bases de dadosSCIELO, MEDLINE e Google Acadêmico.

\begin{tabular}{|c|c|c|c|}
\hline Título & Autor & Ano & Argumentação \\
\hline $\begin{array}{l}\text { New Coronavirus: } \\
\text { (Re)thinking the } \\
\text { care process in } \\
\text { Primary Health } \\
\text { and Nursing. }\end{array}$ & $\begin{array}{l}\text { Andressa Teoli } \\
\text { Nunciaroni; } \\
\text { Fátima } \\
\text { :Teresinha } \\
\text { eScarparo } \\
\text { hCunha; Liliana } \\
\text { hAngel Vargas; } \\
\text { Vanessa de } \\
\text { Almeida } \\
\text { Ferreira } \\
\text { Corrêa. }\end{array}$ & & $\begin{array}{l}\text { Expõe ações específicas da APS no } \\
\text { enfrentamento à COVID-19, como: à } \\
\text { divulgação e implementação de } \\
\text { medidas de controle e de informações } \\
\text { em saúde de qualidade e à } \\
\text { reorganização dos fluxos internos das } \\
\text { unidades. Destaca ainda que é por } \\
\text { meio do protagonismo comunitário e } \\
\text { das redes solidárias que os proces } \\
\text { sosde cuidado de enfermagem serão } \\
\text { (re)criados. }\end{array}$ \\
\hline $\begin{array}{l}\text { ATENÇÃO } \\
\text { PRIMÁRIA } \\
\text { SAÚDE } \\
\text { COMBATE } \\
\text { COVID-19: } \\
\text { IMPORTÂNCIA E } \\
\text { DESAFIOS DOS } \\
\text { PROFISSIONAIS }\end{array}$ & $\begin{array}{l}\text { Maraiza Alves } \\
\text { der Oliveira; } \\
\text { Jéssica Oliveira } \\
\text { da Cunha; Rita } \\
\text { der Cássia } \\
\text { Santar Rosa } \\
\text { Matos; Allan } \\
\text { Dantas dos } \\
\text { Santos; Grace } \\
\text { Anme Azevedo } \\
\text { Dória. }\end{array}$ & & $\begin{array}{l}\text { Apresenta informações que reafirmam } \\
\text { a importância da APS no cenário atual, } \\
\text { através do atendimento resolutivo para } \\
\text { casos leves e identificação precoce } \\
\text { para os casos graves. Além disso, } \\
\text { destaca que os profissionais de saúde } \\
\text { devem utilizar de outras estratégias } \\
\text { para alcançar a resolutividade. }\end{array}$ \\
\hline $\begin{array}{l}\text { Consulta de } \\
\text { Enfermagem aos } \\
\text { casos suspeitos } \\
\text { de COVID -19, na } \\
\text { Atenção Primária } \\
\text { aSaúde }\end{array}$ & $\begin{array}{l}\text { Conceição } \\
\text { eCeanny } \\
\text { sFormiga Sinval } \\
\text { sCavalcante; } \\
\text { aJayra Adrianna } \\
\text { ada Silva Sousa; } \\
\text { Ana Maria de } \\
\text { Araújo Dias. }\end{array}$ & 2020 & $\begin{array}{l}\text { Ressalta que a APS deve ser } \\
\text { considerada um importante pilar frente } \\
\text { a situações emergenciais, sendo } \\
\text { também porta de entrada para os } \\
\text { pacientes suspeitos e/ou confirmados } \\
\text { de Covid-19. Em relação à } \\
\text { Enfermagem, adota papel importante, } \\
\text { pois através de sua consulta de } \\
\text { Enfermagem aplica protocolos } \\
\text { estabelecidos para o momento. }\end{array}$ \\
\hline
\end{tabular}




\begin{tabular}{|c|c|c|}
\hline 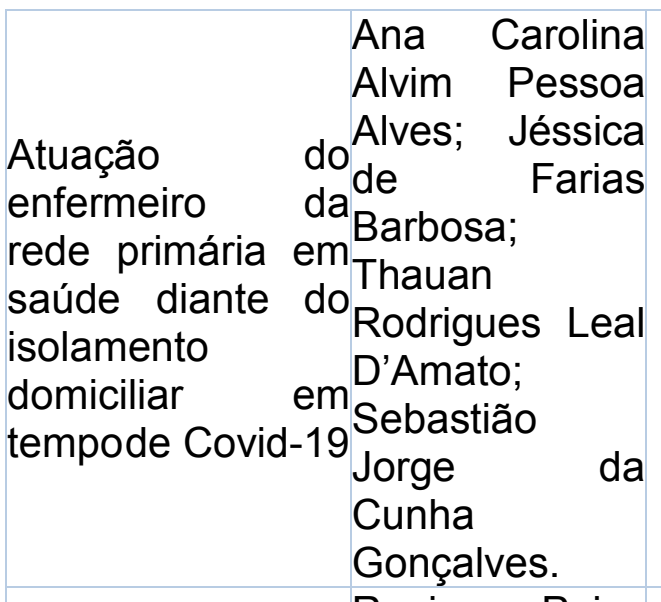 & 2020 & $\begin{array}{l}\text { Apresenta ferramentas utilizadas para } \\
\text { dar continuidade na assistência de } \\
\text { pessoas que necessitam de } \\
\text { monitoramento diário e cuidados } \\
\text { regulares. Além disso, considera que o } \\
\text { enfermeiro deve ter atenção especial } \\
\text { para qualidade e proteção da vida, } \\
\text { integralidade das ações, controle social e } \\
\text { para inclusão da educação em saúde. }\end{array}$ \\
\hline 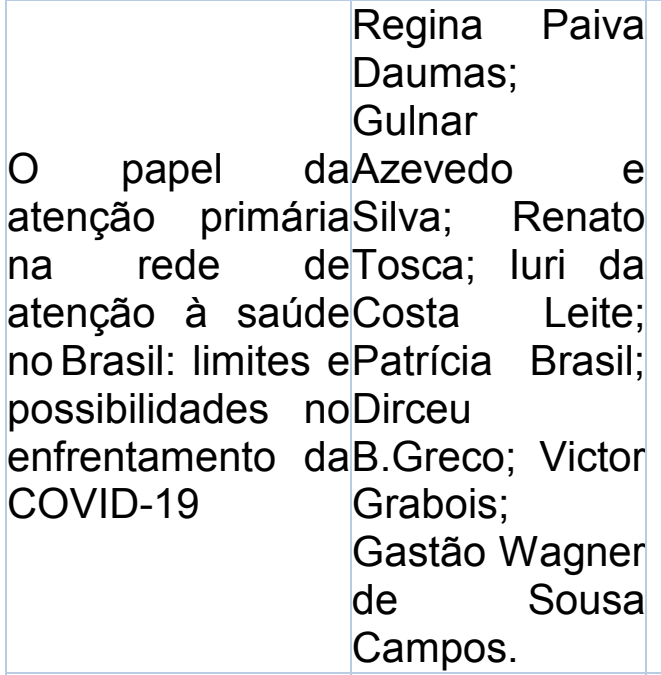 & 2020 & $\begin{array}{l}\text { Relata que o trabalho comunitário pode } \\
\text { atuar para a redução da disseminação da } \\
\text { infecção, acompanhar os casos leves em } \\
\text { isolamento domiciliar, apoiar as } \\
\text { comunidades durante o distanciamento } \\
\text { social, identificare conduzir situações de } \\
\text { vulnerabilidade individual ou coletiva e, } \\
\text { principalmente, garantir o acesso a } \\
\text { cuidados de saúde e o necessário } \\
\text { encaminhamento nas fases mais críticas } \\
\text { da epidemia. }\end{array}$ \\
\hline $\begin{array}{ll} & \begin{array}{l}\text { Rodrigo } \\
\text { Jenses; Rudval }\end{array} \\
\text { Contribuições da } & \text { Souza da Silva; } \\
\text { rede de pesquisa } & \text { Sheila Coelho } \\
\text { em processo de } & \text { Ramalho } \\
\text { enfermagem para } & \text { Masconcelos } \\
\text { assistência } \quad \text { na } & \text { Morais; Tania } \\
\text { pandemia } \quad \text { de } & \text { Machado } \\
\text { COVID-19 } & \text { Chianca; } \\
& \text { Vinicius Batista } \\
& \text { Santos. }\end{array}$ & 2020 & $\begin{array}{l}\text { Expõe a criação de cinco instrumentos } \\
\text { envolvendo os elementos da prática de } \\
\text { enfermagem (diagnósticos, resultados e } \\
\text { intervenções de enfermagem) na } \\
\text { assistência à comunidade e ao paciente } \\
\text { e no suporte à saúde do trabalhador de } \\
\text { enfermagem no contexto da pandemia } \\
\text { de COVID-19 em todo Brasil. }\end{array}$ \\
\hline
\end{tabular}

Fonte: Dados da pesquisa, 2021. 


\section{DISCUSSÃO}

Tendo em vista todos os atributos da Atenção Primária de Saúde, que versam entre: acesso, integralidade, longitudinalidade, coordenação do cuidado, orientação familiar, orientação comunitária e competência cultural; destaca-se a importância de uma organização dos serviços, visando às adaptações necessárias para a garantia de um fluxo operacional durante a pandemia do Coronavírus (ALVES et al., 2020; BRASIL, 2020).

As Unidades de Saúde devem ser organizadas com o objetivo de identificar os possíveis casos de covid-19, classificando-os de acordo com a gravidade dos mesmos. Quando em casos leves, os pacientes devem ser tratados e acompanhados na própria Unidade de Saúde e quando em casos graves, deve-se priorizar a estabilização do paciente e o seu encaminhamento para o serviço de referência. Ainda nesse contexto, a notificação compulsória deve ser realizada pela equipe da unidade de saúde, incluindo a adesão de medidas preventivas que impeçam uma transmissão comunitária (BARBOSA; SILVA, 2020).

Como medida de prevenção para contaminação dos profissionais das unidades de saúde, recomenda-se a realização do teleatendimento, até mesmo quando o paciente forpositivo para Sars-CoV-2. O atendimento presencial na UBS deve ser priorizado para pacientes com queixas agudas não respiratórias e para aqueles que apresentam uma agudização de doenças crônicas, cuja assistência pode ser fornecida na APS, evitando a superlotação de serviços de maior complexidade (DAUMAS et al., 2020). Caso não seja possível a realização do teleatendimento, recomenda-se que haja a visita domiciliar, estandoos profissionais em uso de equipamentos de proteção individual (EPI) (TONIN et al., 2020).

Uma APS organizada, com profissionais qualificados e em número suficiente pode contribuir de maneira significativa para a diminuição da incidência de infecção por parte da população adscrita. Os enfermeiros e demais profissionais que compõem a equipe, devem participar de todos os processos de trabalho na unidade 
de saúde, reconhecendo a importância da atenção especial para a qualidade de assistência fornecida (BARRA et al., 2020; DAUMAS et al., 2020).

Neste cenário, o enfermeiro como gestor e integrante da equipe, atua na ESF com várias atribuições, entre elas assistência integral às pessoas e famílias, realiza consulta de enfermagem, solicitação de exames complementares, prescrição de medicamentos e educação em saúde. Além disso, participa de todos os processos de trabalho no planejamento, organização, execução e avaliação das ações implantadas (ALVES, 2020; CAVALCANTE, 2020).

Ademais, a enfermagem utiliza componentes do método científico para identificar situações de saúde/doença, prescrever e implementar medidas de enfermagem, realiza promoção, prevenção, proteção, recuperação e reabilitação da saúde. Essas ações de enfermagem são práticas regulares na ESF, que possibilitam a identificação precoce dos agravos de saúde, a criação de vínculos com os usuários e o planejamento das ações (CAVALCANTE, 2020).

Diante da pandemia do Covid-19 na APS, despertou a força da Atenção Básica desencadeando em reflexões sobre financiamentos e investimentos. Além disso, estabeleceu- se a força presente na cooperação entre os níveis de atenção e firmando-se a APS como porta de entrada de todo o sistema de saúde e com alto poder de resolutividade (NUNCIARONI, 2020).

No entanto, para uma assistência de qualidade devem ser garantidos os atributos essenciais: acesso, primeiro contato, longitudinalidade e integralidade. Como também, os secundários: orientação familiar, orientação comunitária, competência cultural, visando as demandas especificas do território adscrito (NUNCIARONI, 2020).

Assim, ressalta-se que os serviços de atendimento primário devem assumir papel resolutivo frente aos casos leves, principalmente na identificação precoce, além do rápido encaminhamento dos casos graves, tornando-se uma ferramenta primordial para definir a conduta correta dos pacientes (OLIVEIRA et al.,2020).

$\mathrm{Na}$ Sistematização de Assistência de Enfermagem (SAE) podem se apresentar de forma pouco organizada devido ao conhecimento limitado acerca da COVID-19. Por isso, a necessidade estudos envolvendo a temática para construção de protocolos e documentos com o objetivo do planejamento de intervenções de 
enfermagem. Assim, pretende-se a organizaçãodos serviços de saúde no cenário de enfrentamento do coronavírus (BARROS, 2020).

\section{CONCLUSÃO}

Os medos e incertezas associados ao novo coronavírus gera uma crise emocional/social que exige da população uma adaptação rápida ao novo estilo de vida. Destaca-se que, portanto, a competência ética/política/humanística da enfermagem diante do contexto apresentado. Além disso, a APS é o pilar na redução de sucateamentos em saúde devido a sua atuação de promoção e prevenção de riscos a comunidade.

Portanto, faz-se necessário que os profissionais de saúde protagonizem discussões e mobilizações em torno da defesa do Sistema Único de Saúde público, universal e do fortalecimento da Atenção Primária como porta de entrada do SUS. Além do mais, os governantes do país devem reconhecer o papel dos profissionais e das redes de atenção à saúde através da segurança a saúde do trabalhador, de benefícios as categorias e boas estruturas de cuidado, visto que a pandemia potencializou a fragilidade do sistema de saúdeem vários aspectos, como: falta de recursos materiais e financeiros e óbitos em massa de profissionais.

\section{REFERÊNCIAS BIBLIOGRÁFICAS}

ALVES, Ana Carolina Alvim Pessoa et al. Atuação do enfermeiro da rede primária em saúde diante do isolamento domiciliar em tempo de Covid-19. Revista Pró-univerSUS, v.11, n.2, p.97-101, 2020.2 Disponível em: http://editora.universidadedevassouras.edu.br/index.php/RPU/article/view/2481. Acesso em: 14 fev. 2021.

BARROS, Alba Lúcia Bottura Leite et al. Contribuições da rede de pesquisa em processo de enfermagem para assistência na pandemia de COVID-19. Rev Bras Enferm, v.73, 2020.

Disponível em: https://www.scielo.br/scielo.php?pid=S003471672020001400505\&script=sci_arttext\&tlng=pt. Acesso em: 18 fev. 2021.

CAVALCANTE, Conceição Ceanny Formiga Sinval; SOUSA, Jayra Adrianna da Silva; DIAS, 
Ana Maria de Araújo. Consulta de Enfermagem aos casos suspeitos de COVID -19, naAtenção Primária a Saúde. Revista da FAESF, v. 4, p. 34-40, 2020. Disponível em: https://www.faesfpi.com.br/revista/index.php/faesf/article/view/112. Acesso em: 09 fev. 2021.

DAUMAS, Regina Paiva et al. O papel da atenção primária na rede de atenção à saúde no Brasil: limites e possibilidades no enfrentamento da COVID-19. Cad. Saúde Pública, v. 36,n.6, 2020. Disponível em: https://www.scielosp.org/article/csp/2020.v36n6/e00104120/pt/. Acesso em: 18 fev. 2021.

DAVID, Helena Maria Scherlowski Leal et al. Pandemia, conjunturas de crise e prática profissional: qual o papel da enfermagem diante da Covid-19? Revista Gaúcha de Enfermagem, v. 42, n.42(esp), $2021 . \quad$ Disponível em: https://pesquisa.bvsalud.org/portal/resource/pt/biblio-1139166 Acesso em: 09 fev. 2021.

NUNCIARONI, Andressa Teoli et al. Novo Coronavírus: (re)pensando o processo de cuidadona Atenção Primária à Saúde e a Enfermagem. Rev Bras Enferm, v. 73, 2020. Disponível em: https://pesquisa.bvsalud.org/portal/resource/pt/mdl-32965400 Acesso em: 18 fev. 2021.

OLIVEIRA, Maraiza Alves et al. ATENÇÃO PRIMÁRIA A SAÚDE NO COMBATE A COVID-19: IMPORTÂNCIA E DESAFIOS DOS PROFISSIONAIS. RevIPI, v.7, n.2, p.31-40, 2020. Disponível em: https://www.revista.ufs.br/index.php/revipi/article/view/14221.

Acesso em 11 fev. 2021.

TOCANTIS. Secretaria do Estado da Saúde. NOTA TÉCNICA Nº 01, DE 26 DE MARÇO DE 2020 ORIENTA A ATENÇÃO PRIMÁRIA À SAÚDE DOS MUNICÍPIOS PARA O ENFRENTAMENTO DO NOVO CORONAVÍRUS (COVID-19), p.8, 2020. Disponível em: https://central3.to.gov.br/arquivo/501323/. Acesso em: 14 fev. 2021.

SANCHEZ, Maritza Consuelo Ortiz et al. Pandemia do Coronavírus e Atenção Primária: reflexões sobre os desafios dos gestores. Research, Society and Development, v.9, n.7, 2020. Disponível em: https://www.rsdjournal.org/index.php/rsd/article/view/4154/3468. Acesso em 10 fev. 2021.

SILVA, Manoel Carlos Neri et al. ENFERMAGEM E A PANDEMIA DA COVID-19: UMA CONJUGAÇÃO ENTRE LIDERANÇA E VULNERABILIDADE PROFISSIONAL. Enferm. Foco, v.11, n.2, 2020. Disponível em: https://scholar.google.com.br/scholar?hl=ptBR\&as_sdt $=0 \% 2 C 5 \& q=E N F E R M A G E M+E+A+P A N D E M I A+D A+C O V I D-$

$19 \% 3 A+U M A+C O N J U G A \% C 3 \% 87 \%$ C3\%83O+ENTRE+LIDERAN\%C3\%87A+E+VULN

ERABILIDADE+PROFISSIONAL\&btnG. Acesso em: 14 fev. 2021.

SILVA, Manoel Carlos Neri et al. Enfermagem e a pandemia da Covid-19: uma conjugação entre liderança e vulnerabilidade profissional. Enfermagem em Foco, v. 11, n. 2. ESP, 2020.Disponível em: https://scholar.google.com.br/scholar?hl=ptBR\&as_sdt $=0 \% 2 \mathrm{C} 5 \& \mathrm{q}=.+$ Enfermagem $+\mathrm{e}+\mathrm{a}+$ pandemia $+\mathrm{da}+$ Covid -

$19 \% 3 \mathrm{~A}+$ uma+conjuga $\% \mathrm{C} 3 \% \mathrm{~A} 7 \% \mathrm{C} 3 \% \mathrm{~A} 3 \mathrm{o}+$ entre+lideran $\% \mathrm{C} 3 \% \mathrm{~A} 7 \mathrm{a}+\mathrm{e}+\mathrm{vulnerabilidade}+$ pro fissional\&btnG=. Acesso em: 14 fev. 2021. 